\title{
Maintaining New Zealand's Electrical Reserve Management Tool
}

\author{
A. Prince-Pike*, D. I. Wilson*, I. Ilieva ${ }^{\dagger}, \mathrm{A}^{*} \mathrm{Li}^{\dagger}$ and M. Phethean ${ }^{\dagger}$ \\ *Electrical \& Electronic Engineering \\ Auckland University of Technology, Auckland, New Zealand \\ Email: aprincep@aut.ac.nz \\ †Systems Investigations Group \\ Transpower \\ Wellington, New Zealand
}

\begin{abstract}
Managing the electrical reserves in a small, geographically elongated nation like New Zealand is a challenge. The Reserve Management Tool (RMT) developed over a decade ago optimally schedules the reserve load given the current and anticipated circumstances every half hour to ensure that the reserve requirement for electricity is always met. However with changes in the underlying computing platform, and the recognition that the tool needed to be more flexible to incorporate future generator development meant that this tool needed a revamp. This paper describes the present tool, the motivation for the redesign and demonstrates the new capabilities.
\end{abstract}

\section{INTRODUCTION}

Maintaining the delicate balance between supply and demand in New Zealand's electricity system poses unique challenges to reserve management compared to traditional synchronously interconnected electricity systems due to New Zealand's small size and elongated geography. As illustrated in Fig. 1, New Zealand consists of two main islands, (imaginatively named the North and South Islands), running asynchronous power grids connected via an HVDC bi-pole link. This bi-directional link is critical to maximising resources and maintaining system stability since the less populated South Island possess significant hydro generators.

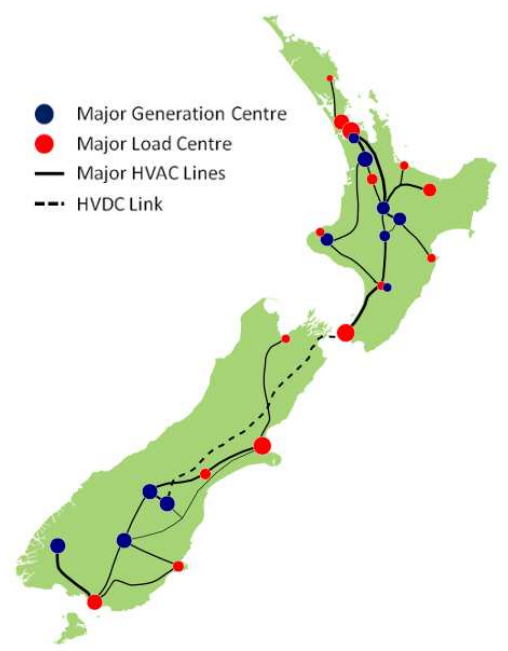

Fig. 1. The High Voltage New Zealand Electricity Transmission System [1]
In large interconnected systems such as in Europe or North America, a single generator represents only a small portion of the total generation, so therefore tripping of a single generator usually only results in a small frequency fluctuation. However in New Zealand and other small systems such as Singapore [2], the larger generating units or the HVDC link represents a significant portion of the total generation and should they trip, will result in a large frequency fluctuation [3, 4].

The Reserve Management Tool developed by Transpower, a New Zealand state owned enterprise and the System Operator, enables the operators to dynamically model the reserve requirements and automatically determine and schedule the required reserve to meet the under frequency targets specified in the regulations.

This paper gives an overview of the Reserve Management Tool (RMT) and describes the important changes made when migrating the software from MATLAB released in 1999 to MATLAB release 2011. The upgrade was necessary to improve the flexibility when adding new generators, when changing frequency standards, and to improve the maintenance of the internal dynamic models. This document describes the changes, and the rationale behind the redesign to the (compiled) MATLAB script RMT_shell and associated files and the (compiled) SIMULINK model RMT_model.

These changes were necessary due to changes in the way MATLAB and SIMULINK transferred information between each other in compiled mode between MATLAB 1999 and 2011. Apart from resolving compatibility issues, this upgrade has made it easier, and less error prone to make updates to the tool such as adding or modifying existing stations. This improves the sustainability of the tool.

\section{The NZ Electricity MARKeT}

The electricity market in New Zealand was deregulated during the 1990s. Transpower, as the System Operator, is responsible for scheduling, pricing and dispatching generation at minimum cost according to market rules. In addition, Transpower is also responsible for providing ancillary services to the market including the management of reserves.

The electricity market operates on a nodal pricing basis where bids from energy providers (generators) and interruptible load offers are loaded into a nodal pricing algorithm called 
SPD (Scheduling, Pricing and Dispatching solver) [5]. SPD uses the generation offers, sheddable load offers, along with information on the HVDC link from the System Operator to create an optimal dispatch schedule every 30 minutes at minimum cost.

In parallel to SPD is the RMT tool which provides an automated process for reserves management within the New Zealand power system. It simulates the system frequency response based on the current optimal dispatch schedule from SPD to determine amount of reserve required to cover the largest single risk in the system before passing the data back to SPD.

Together, SPD and RMT produce a co-optimised dispatch schedule for each trading period which are then sent back to generators and interruptible load providers.

\section{A. Quality obligations}

The Electricity Industry Participation Code [6] defines limits for the frequency excursion that occurs during a contingency event. For a contingent event the frequency must not fall below $48 \mathrm{~Hz}$, for an extended contingent event it must not fall below $47 \mathrm{~Hz}$ in the North Island and $45 \mathrm{~Hz}$ in the South Island.

A Contingent Event (CE) is defined as an event which does not require the disconnection of demand to maintain grid stability. The loss of the largest generator or a single HVDC pole is covered as a CE. An Extended Contingent Event (ECE) involves the disconnection of loads in order to maintain grid stability and typically occurs during the loss of both poles on the HVDC link.

To cover the MW loss from generation, two types of reserves are scheduled, Fast Instantaneous Reserve (FIR) and Sustained Instantaneous Reserve (SIR). FIR is reserve that can respond within 6 seconds and be maintained for at least 60 seconds, while SIR is reserve that can respond within 60 seconds and be maintained for 15 minutes. The FIR arrests the immediate drop in system frequency while the SIR maintains the energy balance until a new dispatch schedule can be produced.

\section{B. Review of similar initiatives}

The management of electricity reserves is not unique to New Zealand and many system operators have modelling tools or approaches to estimate reserve requirements. An extensive review of reserve requirements across eight electrical systems is given in [7]. All the reviewed electricity providers had requirements on FIR and SIR (or equivalent), however the time constants involved were much longer than New Zealand which is due to the small size of the New Zealand system. An interesting observation is that many providers had either fixed amounts of FIR and SIR or simply a fraction of the system load. Taking such an approach simplifies the calculation of the required reserve, but is probably sub-optimal.

Bakken et al in [8] explores reserves management, however they focus on developing a tool to analyse relatively slow power system dynamics in the minutes to hours range.

Renewable energy generation, particularly wind, is becoming increasingly common. The variability of wind makes management of reserves challenging and a concern for system operators. This has let to new probabilistic modelling and forecasting tools to determine reserve requirements $[9,10]$. While Transpower is concerned about wind power uncertainty, New Zealand only has a relativity small wind installation at present and subsequently RMT does not contain dedicated wind forecasting.

The electricity reserve market has been the focus of considerable research [11-13]. However due to the unique under frequency requirements of New Zealand a more rigorous approach is required to determine the amount of reserves.

\section{The Reserve MAnAgEment Tool (RMT)}

The RMT tool was originally developed in 2000 by Roger Miller and colleagues at Transpower to replace the earlier FORTRAN-based RTFCalc (Real Time Frequency calculator) with an automated, detailed dynamic model of the New Zealand power system. RMT was originally developed in a MATLAB release from 1999, [14, 15].

As noted in section II, RMT is only half of the economic optimisation loop. RMT is called by the economic SPD solver every half hour (trading period) with the current optimal dispatch schedule which includes cleared generation, FIR and SIR offerings and HVDC loading. RMT then returns the computed frequency curves assuming the loss of the single largest provider or the HVDC link, and MW loadings for each station, as well as set of Net Free Reserve (NFR) factors. The NFRs are used by SPD to optimally allocate the amount of reserves required across multiple generators in a physically realisable manner at minimum cost. This cyclic process is illustrated in Fig. 2.

The RMT tool consists of two components: a controlling executive collection of MATLAB scripts collectively called the Shell, and a SimULINK dynamic model of the New Zealand power system. The Shell loads the scenario data received from the SPD solver and computes the initial conditions for the dynamic model such as initial gate positions for hydro stations. The resulting simulation data is then passed back to the Shell for post processing and it returns the results back to SPD once a satisfactory solution has been reached.

To ease the deployment of RMT onto Transpower's computing servers and decrease execution time (particularly for the dynamic model), both the Shell and Simulink Model are compiled into executable applications using the MATLAB compiler and Simulink Real Time Workshop toolboxes. This enables deployment on multiple machines without needing extra software licences, however one of the main problems is the data exchange between the Shell and dynamic model. This application is unique because both model structure and the parameters are changing each iteration, not just constants or input data to the dynamic elements. This makes compilation non trivial.

Apart from modelling reserve, RMT is also used for post event analysis. The system frequency during the fault can be loaded into RMT which is used to compare model response against the generator response to an under frequency event. 


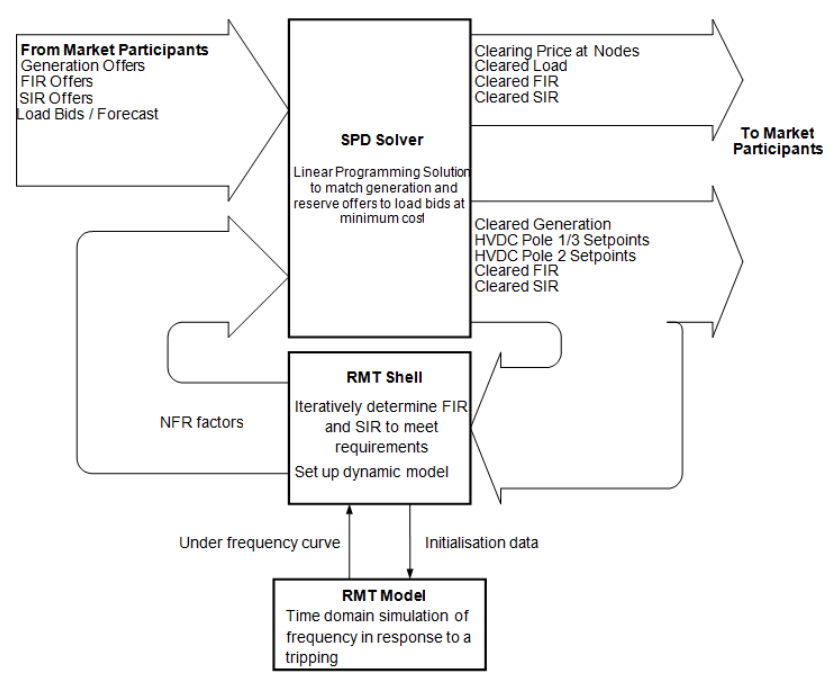

Fig. 2. The role of RMT in the electricity SPD market process. Figure adapted from [14].

\section{A. The RMT executive}

The RMT executive (or Shell) is a collection of MATLAB scripts and functions which load the current dispatch schedule exported from the SPD solver in the form of a flat CSV file. The Shell then calculates the initial conditions for the SIMULINK time domain simulation and configures current operating parameters such as turbine outputs. These conditions are passed to the SIMULINK model using a data MATLAB . mat file. If the frequency response is outside the specifications for either of the islands, through a process of iteration, the Shell adjusts the initial conditions and the process repeats until the correct amount of FIR and SIR are found so that under frequency targets are just met. The shell then returns the NFR factors to the SPD solver which allocates the reserves required in a physically realisable manner to generators which are offering reserve. It is the establishing of the conditions to just meet the under-frequency target that is the main purpose of the RMT tool.

\section{B. SIMULINK model}

Using in-house knowledge and information (required by legislation) from asset owners, Transpower has constructed a detailed model of the New Zealand power system in SIMULINK. The model represents the power balance between generation and load for both islands. Tripping a generator or the HVDC link results in a mismatch between generation and load which leads to a dynamic frequency fluctuation in each island.

RMT represents each island as a two bus system with generators and the HVDC link connected to one bus and load connected to the other bus as illustrated in Fig. 3. Transmission losses are modelled as a resistive load between the buses. The AC networks within each island are not modelled as from a reserve management point of view the topology is irrelevant. This assumption is not valid if reserves cannot be supplied to the load without overloading transmission circuits, however this constraint currently in New Zealand is very rare. It is important to note that RMT is effectively performing a DC simulation as it is not simulating power factors or impedances. Clearly this is a simplification.

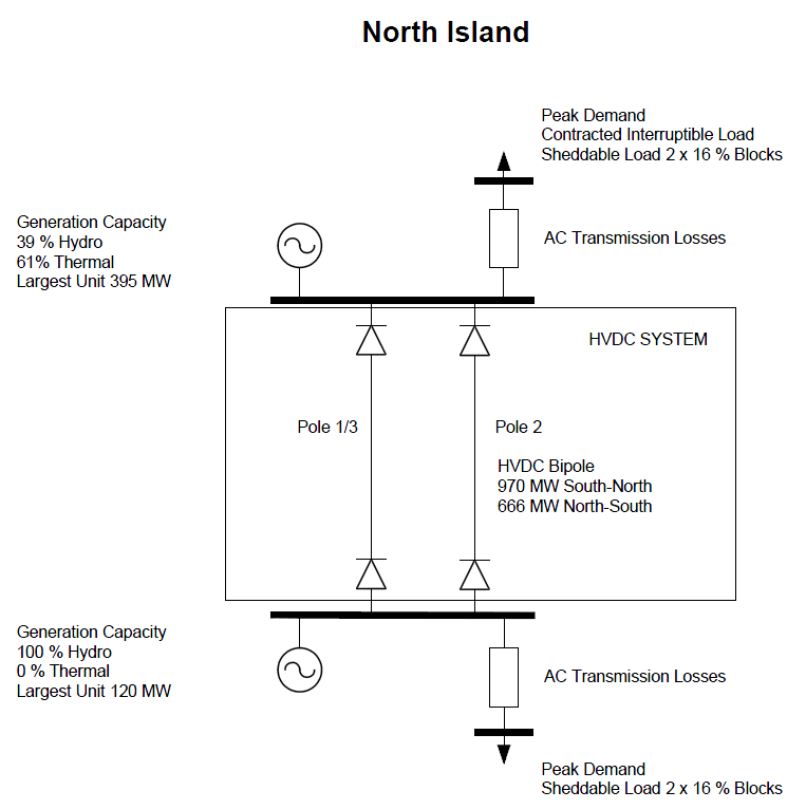

South Island

Fig. 3. New Zealand Electricity System representation [14]

The SImUlinK model is divided into a North Island representation and a South Island representation, connected by the HVDC link as shown in Fig. 4. The dynamic model currently contains models of the following station types: partly loaded hydro, tail water depressed hydro, steam turbine, geothermal, gas turbine, combined cycle gas turbine and ungoverned generator. On the load side there are sheddable load and uncontrolled load models. The two islands are connected via the HVDC bipole model. All generator and load model instances include under, and over, frequency protection relay models which are configured and enabled depending on the station.

The system load is modelled either as individual sheddable load models which include contracted interruptible load and the Automatic Under Frequency Load Shedding Scheme, or a single lumped uncontrolled load in each island.

Each model has a single input which is the frequency deviation normalised to $50 \mathrm{~Hz}$ and two outputs: the accelerated power in MW, and mechanical starting time in seconds. These are summed across each island to find the total accelerating power and mechanical starting time in each island. This accelerating power is the difference between the power injection from stations and off-take by the loads. Fig. 5 gives an overview of the time domain simulation model in SIMULINK.

The elements in the SIMULINK model have been validated using station test data from asset owners and using data from historical under frequency events which resulted in large 


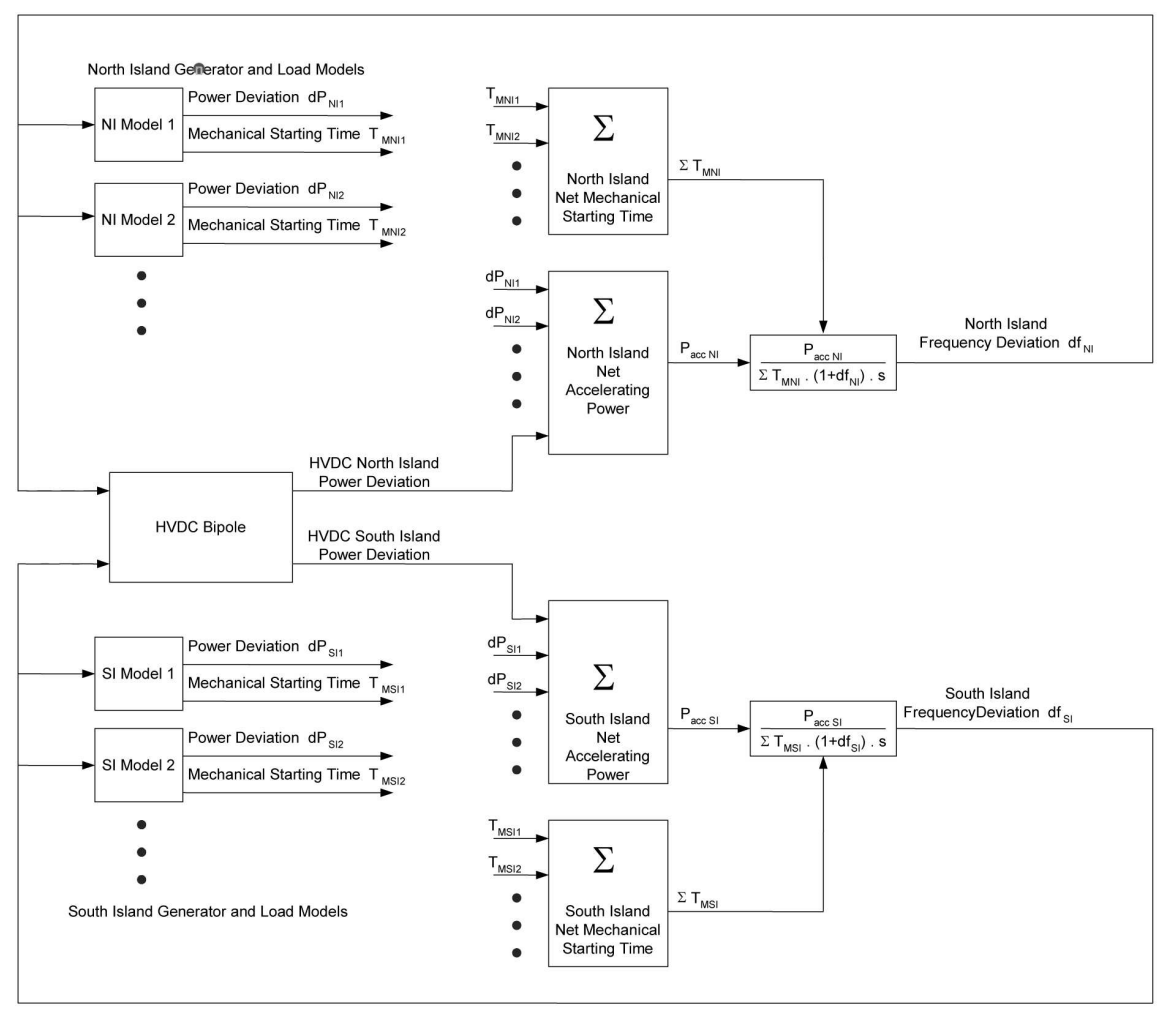

Fig. 5. An overview of the simulation model [16]

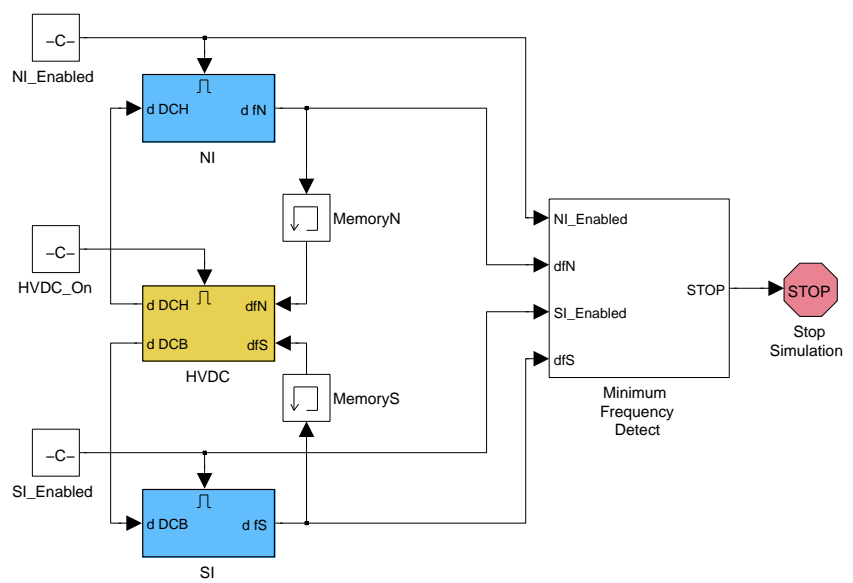

Fig. 4. New Zealand Electricity System representation in SIMULInK

frequency fluctuations.

\section{Data exchange between the compiled applications}

RMT along with the SPD solver are run every 30 minutes on a distributed computing cluster. In order to simplify the process and alleviate the need for a large number of MATLAB licences, RMT has been compiled into an executable file using the MATLAB compiler. A side benefit of compilation is that the dynamic SimUlink model runs an order of magnitude faster when compiled. Unfortunately when compiled, the two separate executables (shell and dynamic model) cannot use the Matlab workspace to exchange variables. So in order to pass the necessary information between the executive and dynamic model, the SIMULINK model is compiled with the Rapid Simulation Target and uses a parameter structure in the form of a . mat file which is dynamically generated by the Matlab executable to the Simulink executable. The structure of the parameter file is determined by the SIMULINK compiler.

This is quite different from the usual compiled SimulinK applications which in say control type applications, are structurally static systems that simply process differing input data streams, and possibly output results. The complication in this instance with RMT is that both the model parameters (such as gains and integrator initial conditions) and model structure are changing at each iteration as well as the input data streams.

\section{$D$. The need for the upgrade}

The primary reason that necessitated the RMT upgrade was the significant change implemented by the Mathworks in the model parameter structure between MATLAB 1999 and 2011. The Matlab 2011 version of the Simulink Real Time Workshop toolbox generates a parameter structure which is significantly different, both in content and structure, from the MATLAB 1999 version. This vector is used by the (compiled) script file RMT_Shell to pass data such as initial conditions to RMT_Model (the SIMULINK model) and is updated each iteration until RMT_Shell arrives at a converged solution.

The old method of data exchange contained a model checksum array, and a double array which is 22,264 elements long 
and contains every parameter and variable in RMT_Model. This includes every lookup table, all PID governor constants for every station, initial gate positions, turbine ratings and so on. With so many variables exchanged and no direct mapping between variables and their location in the variable array it was very difficult to maintain.

\section{BALANCING THE REQUIREMENTS FOR THE TOOL}

Apart from making RMT compatible with MATLAB 2011, the upgrade aims both allow future users to easily add new stations, (of both existing and new types (e.g. tidal stations), and to ease the maintenance and future proof the tool for potential changes in the underlying computing environment.

One of the main modifications to RMT was updating the strategy of modifying dynamic model parameters. Originally there were a number of blank station templates of each type (hydro, geothermal, combined cycle etc). During the simulation RMT Shell would overwrite every parameter contained in every model instance RMT Model to customise the templates to each station that was active. This involved managing and passing over 22,000 parameters between RMT Shell and RMT Model. An immediate concern was that the maximum number of hydro stations (30 in the original model), was rapidly becoming a constraint.

To reduce the number of parameters and make the program maintainable, station templates are no longer used. Each station has been named and constant parameters specific to that station are hard coded in the model mask in the SIMULINK diagram, so only the parameters which change each iteration are passed. If a station is not active for a particular scenario it is disabled and plays no part in the simulation. This has reduced the number of variables to around 1000. This resulted in a small speed increase, but more importantly improved code maintainability.

\section{RESUlts}

This section illustrates some of the results generated by RMT using real data from a dispatch record from 5 am on the 18th of January 2012. At this time, the total generation in the North Island was 2286.4MW and 1351.1MW in the South Island. The largest generators were a combined cycle generator producing $260 \mathrm{MW}$ and a hydroelectric generator producing 121MW respectively, with HVDC link transferring 166.3MW of power north.

Fig. 6 shows the RMT tool in action. By the 9th iteration (solid blue line in Fig. 6), RMT has solved for the initial conditions to just keep the frequency above the $48 \mathrm{~Hz}$ limit for the North Island. A similar process is repeated for the South Island, which converged in 3 iterations.

For this scenario, to cover the risk generators identified above, RMT calculated that the amount of FIR required for the North Island is $82.84 \mathrm{MW}$ and $86.16 \mathrm{MW}$ for the South Island. Additional information for the ECE tipping of the HVDC link is also calculated before the data is returned to SPD.

During the course of the upgrade, both MATLAB and fundamental modelling issues were encountered. The MATLAB issues mostly pertained to function and feature depreciation.
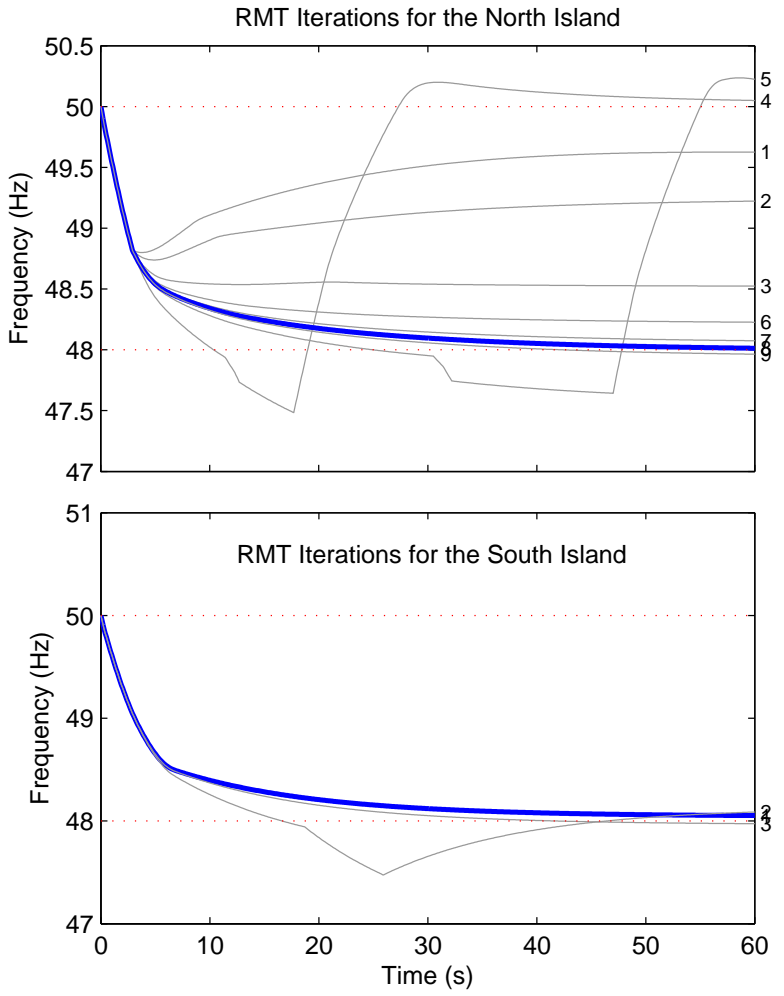

Fig. 6. The predicted frequency excursions in the North and South Island as a function of various initial conditions.

\section{A. Handling race conditions}

All of the stations modelled in the SIMULINK model contain over, and under frequency relay models. These include the combined cycle generators for under frequency protection and hydro stations which use them to modify the feedback loop to improve their response on detection of an under frequency event. Other stations have them but they are currently disabled. Each of these relays has a configurable trip frequency, guard time and inherent delay.

One of the original limitations of compiling SIMULINK applications with the SIMULINK coder is that a fixed step size is necessary. This introduced issues with the original implementation of the under and over frequency relay blocks present which would trip between 0 and 2 time steps later than intended.

\section{B. Future possibilities}

Recently, MATLAB has enabled the use of variable step size integrators in compiled applications. Prior to 2011 only fixed step integrators could be used in a compiled SIMULINK application. Experience has shown that using a variable step size integrator eliminates the race condition problem described earlier but unfortunately the SPD solver and support applications which use the data generated by RMT expect a fixed time step. A second, more sophisticated possibility is to model the full AC network including transmission line effects. Clearly this places a much higher computation load while potentially 
only delivering marginal benefits, especially considering that at present the network is only rarely congested.

\section{CONCLUSIONS}

This paper provides an overview of RMT and describes upgrade of the tool to improve maintainability and ensure compatibility with new releases of MATLAB. The upgraded RMT platform was introduced into the electricity market system in August 2011 and is currently performing as intended.

\section{ACKNOWLEDGEMENTS:}

The authors would like to acknowledge Transpower New Zealand for their contribution to the content in this paper.

\section{REFERENCES}

[1] L. Mortensen, "New Zealand Transmission Grid," 2012.

[2] F. Lu and H. Gan, "National Electricity Market of Singapore," in The 7th International Power Engineering Conference, (IPEC), 29 Nov-2 Dec. 2005, pp. 10071012.

[3] N.-M. Vong, M. Phethean, and S. Nutt, "Managing system frequency dynamics in small power system by hydro generating unit operated in Tail Water Depress (TWD) mode with use of Feed Forward Controls," in Power and Energy Society General Meeting, (IEEE), July 2010, pp. $1-8$.

[4] N.-M. Vong, J.-S. Tan, S. Nutt, and P. Vaughan, "Feedforward control on hydro generating units as spinning reserve: New Zealand's experience of the effect on postevent frequency regulation," in 18th Conference of the Electronic Power Supply Industry, 2010.

[5] T. Alvey, D. Goodwin, X. Ma, D. Streiffert, and D. Sun, "A Security-Constrained Bid-Clearing Sysyem for the New Zealand Wholesale Electricity Market," IEEE Transactions on Power Systems, vol. 13, no. 2, pp. 340-346, 1998.

[6] Anon, "Electricity Authority - The Act, Code and Regulations," Electricity Authority of New Zealand, Tech. Rep., 2010. [Online]. Available: www.ea.govt.nz/act-code-regs/
[7] Y. Rebours and D. Kirschen, "A survey of definitions and specifications of reserve services," University of Manchester, Tech. Rep., 2005.

[8] B. H. Bakken, A. Petterteig, E. Haugan, and B. Walther, "Stepwise power flow - A new tool to analyse capacity shortage and reserve requirements," in Power Systems Computation Conference (PSCC), Liege, Belgium, 2005.

[9] M. Matos and R. Bessa, "Setting the operating reserve using probabilistic wind power forecasts," IEEE Transactions on Power Systems, vol. 26, no. 2, pp. 594-603, May 2011.

[10] R. Doherty and M. O’Malley, “A new approach to quantify reserve demand in systems with significant installed wind capacity," IEEE Transactions on Power Systems, vol. 20, no. 2, pp. 587-595, May 2005.

[11] K. Cheung, P. Shamsollahi, D. Sun, J. Milligan, and M. Potishnak, "Energy and ancillary service dispatch for the interim ISO New England electricity market," IEEE Transactions on Power Systems, vol. 15, no. 3, pp. 968974, Aug 2000.

[12] X. Ma, D. Sun, and K. Cheung, "Energy and reserve dispatch in a multi-zone electricity market," IEEE Transactions on Power Systems, vol. 14, no. 3, pp. 913-919, Aug. 1999.

[13] J. Wang, X. Wang, and Y. Wu, "Operating reserve model in the power market," IEEE Transactions on Power Systems, vol. 20, no. 1, pp. 223 - 229, Feb. 2005.

[14] R. Donaldson, R. Miller, and I. Ilieva, "Reserve Management Tool (RMT) Specification V20," Transpower, New Zealand, Tech. Rep., 2012. [Online]. Available: http://www.ea.govt.nz/industry/mo-serviceproviders/system-operator-market-operation-serviceprovider/

[15] Systems Operation Group, "Reserve management concepts," Transpower, New Zealand, Tech. Rep., 2012. [Online]. Available: http://www.transpower.co.nz/presentations/rmtanimation/

[16] R. Miller and R. Hardy, "Managing Frequency Performance in the Deregulated Electricity Market of NZ," Transpower, New Zealand, Tech. Rep., 2002. 